\title{
MORE SUPPLEMENTS TO A CLASS OF LOGARITHMICALLY COMPLETELY MONOTONIC FUNCTIONS ASSOCIATED WITH THE GAMMA FUNCTION
}

\author{
SENLIN GUO AND FENG QI
}

\begin{abstract}
In this article, a necessary and sufficient condition and a necessary condition are established for a function involving the gamma function to be logarithmically completely monotonic on $(0, \infty)$. As applications of the necessary and sufficient condition, some inequalities for bounding the psi and polygamma functions and the ratio of two gamma functions are derived.

This is a continuator of the paper [12].
\end{abstract}

\section{INTRODUCTION}

Recall $[3,23]$ that a positive function $f$ is said to be logarithmically completely monotonic on an interval $I$ if $f$ has derivatives of all orders on $I$ and

$$
(-1)^{n}[\ln f(x)]^{(n)} \geq 0 .
$$

This kind of functions has very closer relationships with the Laplace transforms, Stieltjes transforms and infinitely divisible completely monotonic functions. For more detailed information, please refer to $[4,8,11,12,20,21,24]$ and related references therein.

It is well-known that the classical Euler gamma function is defined for $x>0$ by

$$
\Gamma(z)=\int_{0}^{\infty} t^{x-1} e^{-t} \mathrm{~d} t
$$

The logarithmic derivative of $\Gamma(z)$, denoted by $\psi(z)=\frac{\Gamma^{\prime}(z)}{\Gamma(z)}$, is called the psi function, and $\psi^{(k)}$ for $k \in \mathbb{N}$ are called the polygamma functions.

For $\alpha \in \mathbb{R}$ and $\beta \geq 0$, define

$$
f_{\alpha, \beta, \pm 1}(x)=\left[\frac{e^{x} \Gamma(x+\beta)}{x^{x+\beta-\alpha}}\right]^{ \pm 1}, \quad x \in(0, \infty) .
$$

The investigation of the function $f_{\alpha, \beta, \pm 1}(x)$ has a long history. In what follows, we would like to give a short survey in the literature.

In $[15$, Theorem 1$]$, for showing

$$
\frac{b^{b-1}}{a^{a-1}} e^{a-b}<\frac{\Gamma(b)}{\Gamma(a)}<\frac{b^{b-1 / 2}}{a^{a-1 / 2}} e^{a-b}
$$

for $b>a>1$, monotonic properties of the functions $\ln f_{\alpha, 0,+1}(x)$ and $\ln f_{\alpha, 0,+1}(x)$ on $(1, \infty)$ were obtained.

In [17, Theorem 2.1], the function $f_{\alpha, 0,+1}(x)$ for $\alpha \leq 1$ was proved to be logarithmically completely monotonic on $(0, \infty)$. In $[13$, Theorem 2.1$]$, the functions

2000 Mathematics Subject Classification. Primary 33B15, 26A48, 26A51; Secondary 26D20, $65 \mathrm{R} 10$.

Key words and phrases. Necessary and sufficient condition, logarithmically completely monotonic function, inequality, ratio, gamma function, psi function, polygamma function, application.

The second author was supported partially by the China Scholarship Council.

This paper was typeset using $\mathcal{A} \mathcal{M} \mathcal{S}$-IAT $\mathrm{E}$ X. 
$f_{\alpha, 0,+1}(x)$ and $f_{\alpha, 0,-1}(x)$ were proved to be logarithmically completely monotonic on $(0, \infty)$ if and only if $\alpha \leq \frac{1}{2}$ and $\alpha \geq 1$ respectively. These results were mentioned in [14, Theorem 2.1] later. However, we do not think the proof in [13] for the necessity is convincible.

In $\left[2\right.$, Theorem 3.2], it was recovered that the function $f_{1 / 2,0,+1}(x)$ is decreasing and logarithmically convex from $(0, \infty)$ onto $(\sqrt{2 \pi}, \infty)$ and that the function $f_{1,0,+1}(x)$ is increasing and logarithmically concave from $(0, \infty)$ onto $(1, \infty)$.

In $\left[1\right.$, p. 376, Theorem 2], it was presented that the function $f_{\alpha, 0,+1}(x)$ is decreasing on $(c, \infty)$ for $c \geq 0$ if and only if $\alpha \leq \frac{1}{2}$ and increasing on $(c, \infty)$ if and only if

$$
\alpha \geq \begin{cases}c[\ln c-\psi(c)] & \text { if } c>0, \\ 1 & \text { if } c=0 .\end{cases}
$$

The necessary and sufficient conditions for the functions $f_{\alpha, 0,+1}(x)$ and $f_{\alpha, 0,-1}(x)$ to be logarithmically completely monotonic on $(0, \infty)$, stated in $[13$, Theorem 2.1$]$ and [14, Theorem 2.1] and mentioned above, were recovered in [5, Theorem 2]. Moreover, the function $f_{\alpha, \beta,+1}(x)$ was proved in [5, Theorem 1] to be logarithmically completely monotonic on $(0, \infty)$ if $2 \alpha \leq 1 \leq \beta$. Using monotonic properties of the functions $f_{1 / 2,0,+1}(x)$ and $f_{1,0,-1}(x)$, the inequality (4) was extended in [5, Remark 1] from $b>a>1$ to $b>a>0$.

In [25], after proving once again the logarithmically completely monotonic property of the functions $f_{1 / 2,0,+1}(x)$ and $f_{1,0,-1}(x)$, in virtue of Jensen's inequality for convex functions, the upper and lower bounds were established: For positive numbers $x$ and $y$, the inequality

$$
\frac{x^{x-1 / 2} y^{y-1 / 2}}{[(x+y) / 2]^{x+y-1}} \leq \frac{\Gamma(x) \Gamma(y)}{[\Gamma((x+y) / 2)]^{2}} \leq \frac{x^{x-1} y^{y-1}}{[(x+y) / 2]^{x+y-2}}
$$

holds, where the middle term in (6) is called Gurland's ratio [16]. The left-hand side inequality in (6) is same as the corresponding one in [16, Theorem 1], but their upper bounds do not include each other.

Recently, some new conclusions on logarithmically completely monotonic properties of the function $f_{\alpha, \beta,+1}(x)$ were procured in [12, Theorem 1]:

(1) If $\beta \in(0, \infty)$ and $\alpha \leq 0$, then $f_{\alpha, \beta,+1}(x)$ is logarithmically completely monotonic on $(0, \infty)$;

(2) If $\beta \in(0, \infty)$ and $f_{\alpha, \beta,+1}(x)$ is a logarithmically completely monotonic function on $(0, \infty)$, then $\alpha \leq \min \left\{\beta, \frac{1}{2}\right\}$;

(3) If $\beta \geq 1$, then $f_{\alpha, \beta,+1}(x)$ is logarithmically completely monotonic on $(0, \infty)$ if and only if $\alpha \leq \frac{1}{2}$.

As direct consequences of these monotonic properties, it is deduced immediately that if $x$ and $y$ are positive numbers with $x \neq y$, then

(1) the inequality

$$
\frac{\Gamma(x+\beta)}{\Gamma(y+\beta)}<\frac{x^{x+\beta-\alpha}}{y^{y+\beta-\alpha}} e^{1 /(y-x)}
$$

for $\beta \geq 1$ and $x>y>0$ holds true if and only if $\alpha \leq \frac{1}{2}$;

(2) the inequality (16) for $\beta \in(0, \infty)$ holds true also if $\alpha \leq 0$.

In this paper, for continuing the work in the paper [12], we consider logarithmically completely monotonic properties of the function $f_{\alpha, \beta,-1}(x)$ on $(0, \infty)$.

The main results of this paper are as follows.

Theorem 1. If the function $f_{\alpha, \beta,-1}(x)$ is logarithmically completely monotonic on $(0, \infty)$, then either $\beta>0$ and $\alpha \geq \max \left\{\beta, \frac{1}{2}\right\}$ or $\beta=0$ and $\alpha \geq 1$. 
Theorem 2. If $\beta \geq \frac{1}{2}$, the necessary and sufficient condition for the function $f_{\alpha, \beta,-1}(x)$ to be logarithmically completely monotonic on $(0, \infty)$ is $\alpha \geq \beta$.

As the first application of Theorem 2, the following inequalities are derived by using logarithmically completely monotonic properties of the function $f_{\alpha, \beta, \pm 1}(x)$ on $(0, \infty)$.

Theorem 3. Let $\beta$ be a positive number.

(1) For $k \in \mathbb{N}$, double inequalities

$$
\ln x-\frac{1}{x} \leq \psi(x) \leq \ln x-\frac{1}{2 x}
$$

and

$$
\frac{(k-1) !}{x^{k}}+\frac{k !}{2 x^{k+1}} \leq(-1)^{k+1} \psi^{(k)}(x) \leq \frac{(k-1) !}{x^{k}}+\frac{k !}{x^{k+1}}
$$

hold in $(0, \infty)$.

(2) When $\beta>0$, inequalities

$$
\psi(x+\beta) \leq \ln x+\frac{\beta}{x}
$$

and

$$
(-1)^{k} \psi^{(k-1)}(x+\beta) \geq \frac{(k-2) !}{x^{k-1}}-\frac{\beta(k-1) !}{x^{k}}
$$

hold on $(0, \infty)$ for $k \geq 2$.

(3) When $\beta \geq \frac{1}{2}$, inequalities

$$
\psi(x+\beta) \geq \ln x \quad \text { and } \quad(-1)^{k} \psi^{(k-1)}(x+\beta) \leq \frac{(k-2) !}{x^{k-1}}
$$

hold on $(0, \infty)$ for $k \geq 2$.

(4) When $\beta \geq 1$, inequalities

$$
\psi(x+\beta) \leq \ln x+\frac{\beta-1 / 2}{x}
$$

and

$$
(-1)^{k} \psi^{(k-1)}(x+\beta) \geq \frac{(k-2) !}{x^{k-1}}-\frac{(\beta-1 / 2)(k-1) !}{x^{k}}
$$

holds on $(0, \infty)$ for $k \geq 2$.

As the second application of Theorem 2, the following inequalities are derived by using logarithmically convex properties of the function $f_{\alpha, \beta, \pm 1}(x)$ on $(0, \infty)$.

Theorem 4. Let $n \in \mathbb{N}, x_{k}>0$ for $1 \leq k \leq n, p_{k} \geq 0$ satisfying $\sum_{k=1}^{n} p_{k}=1$. If either $\beta>0$ and $\alpha \leq 0$ or $\beta \geq 1$ and $\alpha \leq \frac{1}{2}$, then

$$
\frac{\prod_{k=1}^{n}\left[\Gamma\left(x_{k}+\beta\right)\right]^{p_{k}}}{\Gamma\left(\sum_{k=1}^{n} p_{k} x_{k}+\beta\right)} \geq \frac{\prod_{k=1}^{n} x_{k}^{p_{k}\left(x_{k}+\beta-\alpha\right)}}{\left(\sum_{k=1}^{n} p_{k} x_{k}\right)^{\sum_{k=1}^{n} p_{k} x_{k}+\beta-\alpha} .}
$$

If $\alpha \geq \beta \geq \frac{1}{2}$, then the inequality (15) reverses.

As the final application of Theorem 2, the following inequality may be derived by using the decreasingly monotonic property of the function $f_{\alpha, \beta,-1}(x)$ on $(0, \infty)$.

Theorem 5. If $\alpha \geq \beta \geq \frac{1}{2}$, then

$$
I(x, y)<\left[\left(\frac{x}{y}\right)^{\alpha-\beta} \frac{\Gamma(x+\beta)}{\Gamma(y+\beta)}\right]^{1 /(x-y)}
$$


holds true for $x, y \in(0, \infty)$ with $x \neq y$, where

$$
I(a, b)=\frac{1}{e}\left(\frac{b^{b}}{a^{a}}\right)^{1 /(b-a)}
$$

for $a>0$ and $b>0$ with $a \neq b$ is the identric or exponential mean.

Remark 1. For $\beta \in \mathbb{R}$, let

$$
h_{\beta, \pm 1}(x)=\left[\frac{e^{x} \Gamma(x+1)}{(x+\beta)^{x+\beta}}\right]^{ \pm 1}
$$

on $(\max \{0,-\beta\}, \infty)$. In $[10,11]$, it was showed that the functions $h_{\beta,+1}(x)$ and $h_{\beta,-1}(x)$ are logarithmically completely monotonic if and only if $\beta \geq 1$ and $\beta \leq \frac{1}{2}$ respectively. As consequences of monotonic results of the function $h_{\beta, \pm 1}(x)$, the following two-sided inequality was derived in [11]:

$$
\frac{(x+1)^{x+1}}{(y+1)^{y+1}} e^{y-x}<\frac{\Gamma(x+1)}{\Gamma(y+1)}<\frac{(x+1 / 2)^{x+1 / 2}}{(y+1 / 2)^{y+1 / 2}} e^{y-x}
$$

for $y>x>0$, where the constants 1 and $\frac{1}{2}$ in the very left and the very right sides of the two-sided inequality (19) cannot be replaced, respectively, by smaller and larger numbers.

Remark 2. In [9], it was showed that the function

$$
h(x)=\frac{e^{x} \Gamma(x)}{x^{x[1-\ln x+\psi(x)]}}
$$

on $(0, \infty)$ has a unique maximum $e$ at $x=1$, with the following two limits

$$
\lim _{x \rightarrow 0^{+}} h(x)=1 \text { and } \lim _{x \rightarrow \infty} h(x)=\sqrt{2 \pi} .
$$

As consequences of the monotonicity of $h(x)$, it was concluded in [9] that the following inequality

$$
\frac{x^{x[\ln x-\psi(x)-1]}}{y^{y[\ln y-\psi(y)-1]}} e^{y-x}<\frac{\Gamma(y)}{\Gamma(x)}
$$

holds true for $y>x \geq 1$. If $0<x<y \leq 1$, the inequality (22) is reversed.

Remark 3. It is worthwhile to point out that [20, Thorem 1.3] is equivalent to necessary and sufficient conditions in [5, Theorem 2] and [13, Theorem 2.1] for the functions $f_{1 / 2,0,+1}(x)$ and $f_{1,0,-1}(x)$ to be logarithmically completely monotonic on $(0, \infty)$. See also $[11,12]$ and related references therein.

\section{Proofs of Main Results}

Now we are in a position to prove our theorems.

Proof of Theorem 1. Suppose that $f_{\alpha, \beta,-1}(x)$ is logarithmically completely monotonic on $(0, \infty)$. Then

$$
\left[\ln f_{\alpha, \beta,-1}(x)(x)\right]^{\prime}=\ln x-\psi(x+\beta)+\frac{\beta-\alpha}{x} \leq 0,
$$

from which we have

$$
\beta-\alpha \leq x[\psi(x+\beta)-\ln x], \quad x \in(0, \infty) .
$$

If $\beta>0$, then

$$
\beta-\alpha \leq \lim _{x \rightarrow 0^{+}}[x \psi(x+\beta)-x \ln x]=0 .
$$

That is

$$
\alpha \geq \beta .
$$


Using the asymptotic formula

$$
\psi(x)=\ln x-\frac{1}{2 x}+O\left(\frac{1}{x^{2}}\right), \quad x \rightarrow \infty,
$$

see [6, p. 47], in (24) for $\beta>0$, we obtain

$$
\begin{aligned}
\beta-\alpha & \leq \lim _{x \rightarrow \infty} x\left[\ln (x+\beta)-\frac{1}{2(x+\beta)}+O\left(\frac{1}{x^{2}}\right)-\ln x\right] \\
& =\lim _{x \rightarrow \infty}\left[x \ln \left(1+\frac{\beta}{x}\right)\right]-\frac{1}{2} \\
& =\beta \lim _{x \rightarrow \infty}\left[\frac{x}{\beta} \ln \left(1+\frac{\beta}{x}\right)\right]-\frac{1}{2} \\
& =\beta-\frac{1}{2}
\end{aligned}
$$

from which we get

$$
\alpha \geq \frac{1}{2}
$$

Combining (25) and (27) yields

$$
\alpha \geq \max \left\{\beta, \frac{1}{2}\right\} \quad \text { if } \beta>0 .
$$

If $\beta=0$, considering $f_{\alpha, 0,-1}(x)=f_{\alpha, 1,-1}(x)$ and (28) yields $\alpha \geq \max \left\{1, \frac{1}{2}\right\}=1$. The proof is complete.

Proof of Theorem 2. By Theorem 1, the necessary condition is obtained readily.

Differentiating (23) and making use of

$$
\psi^{(n)}(x)=(-1)^{n+1} \int_{0}^{\infty} \frac{t^{n}}{1-e^{-t}} e^{-x t} \mathrm{~d} t, \quad x \in(0, \infty)
$$

and

see [7, p. 884], gives

$$
\frac{1}{x^{n}}=\frac{1}{\Gamma(n)} \int_{0}^{\infty} t^{n-1} e^{-x t} \mathrm{~d} t, \quad x \in(0, \infty),
$$

$$
\begin{aligned}
& (-1)^{n}\left[\ln f_{\alpha, \beta,-1}(x)\right]^{(n)}=\frac{(n-2) !}{x^{n-1}}-(-1)^{n} \psi^{(n-1)}(x+\beta)-\frac{(\beta-\alpha)(n-1) !}{x^{n}} \\
& =\int_{0}^{\infty} t^{n-2} e^{-x t} \mathrm{~d} t-\int_{0}^{\infty} \frac{t^{n-1}}{1-e^{-t}} e^{-(x+\beta) t} \mathrm{~d} t-(\beta-\alpha) \int_{0}^{\infty} t^{n-1} e^{-x t} \mathrm{~d} t \\
& =\int_{0}^{\infty}\left[\alpha-\beta-\frac{1}{t}\left(e^{(1-\beta) t} \frac{t}{e^{t}-1}-1\right)\right] t^{n-1} e^{-x t} \mathrm{~d} t
\end{aligned}
$$

for $n \geq 2$. The inequality

$$
\frac{t}{e^{t}-1}<\frac{1}{e^{t / 2}}, \quad t \in(0, \infty)
$$

was ever used in $[18,19,22]$. Substituting it into (29) leads to

$$
(-1)^{n}\left[\ln f_{\alpha, \beta,-1}(x)\right]^{(n)} \geq \int_{0}^{\infty}\left[\alpha-\beta-\frac{e^{(1 / 2-\beta) t}-1}{t}\right] t^{n-1} e^{-x t} \mathrm{~d} t, \quad n \geq 2 .
$$

Since the function

is increasing, if $\alpha \geq \beta \geq \frac{1}{2}$, then

$$
\frac{e^{(1 / 2-\beta) t}-1}{t}, \quad t \in(0, \infty)
$$

$$
(-1)^{n}\left[\ln f_{\alpha, \beta,-1}(x)\right]^{(n)} \geq(\alpha-\beta) \int_{0}^{\infty} t^{n-1} e^{-x t} \mathrm{~d} t \geq 0, \quad n \geq 2 .
$$


By using (26), it follows that

$$
\left[\ln f_{\alpha, \beta,-1}(x)(x)\right]^{\prime}=\ln \left(1+\frac{\beta}{x}\right)-\frac{1}{2(x+\beta)}+\frac{\alpha-\beta}{x}+O\left(\frac{1}{x^{2}}\right)
$$

as $x \rightarrow \infty$, thus for all $\alpha$ and $\beta$,

$$
\lim _{x \rightarrow \infty}\left[\ln f_{\alpha, \beta,-1}(x)(x)\right]^{\prime}=0 .
$$

From (33) and (32), we have

$$
\left[\ln f_{\alpha, \beta,-1}(x)\right]^{\prime} \leq 0 \quad \text { if } \alpha \geq \beta \geq \frac{1}{2} .
$$

Thus $(-1)^{n}\left[\ln f_{\alpha, \beta,-1}(x)\right]^{(n)} \geq 0$ are valid for all $n \in \mathbb{N}$. The proof is complete.

Proof of Theorem 3. If $f_{\alpha, \beta,-1}(x)$ is logarithmically completely monotonic on $(0, \infty)$, then

$$
(-1)^{k}\left[\ln f_{\alpha, \beta,-1}(x)\right]^{(k)} \geq 0
$$

on $(0, \infty)$ for $k \in \mathbb{N}$, which is equivalent to

$$
\psi(x+\beta) \geq \ln x+\frac{\beta-\alpha}{x}
$$

and, for $k \geq 2$,

$$
(-1)^{k} \psi^{(k-1)}(x+\beta) \leq \frac{(k-2) !}{x^{k-1}}-\frac{(\beta-\alpha)(k-1) !}{x^{k}} .
$$

Hence, Theorem 2 implies inequalities in (12).

If $\beta \geq 1$, Theorem 1 in [12] said that the function $f_{\alpha, \beta,+1}(x)$ is logarithmically completely monotonic on $(0, \infty)$ if and only if $\alpha \leq \frac{1}{2}$, this means that inequalities in (34) and (35) are reversed, and so inequalities in (13) and (14) are valid.

If $\beta>0$ and $\alpha \leq 0$, Theorem 1 in [12] also said that the function $f_{\alpha, \beta,+1}(x)$ is logarithmically completely monotonic on $(0, \infty)$, this means that inequalities in (34) and (35) are also reversed, and so inequalities (10) and (11) are valid.

When $\beta=0$, several mathematicians have proved that the functions $f_{\alpha, 0,+1}(x)$ and $f_{\alpha, 0,-1}(x)$ are logarithmically completely monotonic on $(0, \infty)$ if and only if $\alpha \leq \frac{1}{2}$ and $\alpha \geq 1$ respectively, which implies by reasoning as above the double inequalities (8) and (9). The proof of Theorem 3 is complete.

Proof of Theorem 4. The first conclusion in [12, Theorem 1] implies that the function $f_{\alpha, \beta,+1}(x)$ is logarithmically convex for $\beta>0$ and $\alpha \leq 0$ on $(0, \infty)$. Combining this with Jensen's inequality for convex functions yields

$$
\ln \frac{\exp \left(\sum_{k=1}^{n} p_{k} x_{k}\right) \Gamma\left(\sum_{k=1}^{n} p_{k} x_{k}+\beta\right)}{\left(\sum_{k=1}^{n} p_{k} x_{k}\right)^{\sum_{k=1}^{n} p_{k} x_{k}+\beta-\alpha}} \leq \sum_{k=1}^{n} p_{k} \ln \frac{\exp \left(x_{k}\right) \Gamma\left(x_{k}+\beta\right)}{x_{k}^{x_{k}+\beta-\alpha}},
$$

where $n \in \mathbb{N}, x_{k}>0$ for $1 \leq k \leq n, p_{k} \geq 0$ satisfying $\sum_{k=1}^{n} p_{k}=1, \beta>0$ and $\alpha \leq 0$. Rearranging it leads to the inequality (15).

The third conclusion in [12, Theorem 1] implies that the function $f_{\alpha, \beta,+1}(x)$ is also logarithmically convex for $\beta \geq 1$ and $\alpha \leq \frac{1}{2}$ on $(0, \infty)$, hence, the inequality (36) is also valid for $\beta \geq 1$ and $\alpha \leq \frac{1}{2}$.

Theorem 2 above implies that the function $f_{\alpha, \beta,+1}(x)$ is logarithmically concave for $\alpha \geq \beta \geq \frac{1}{2}$ on $(0, \infty)$, therefore, the inequality (36) is reversed. Theorem 4 is proved.

Proof of Theorem 5. Theorem 2 implies that the function $f_{\alpha, \beta,-1}(x)$ is decreasing on $(0, \infty)$ if $\alpha \geq \beta \geq \frac{1}{2}$, this is,

$$
\frac{e^{y} \Gamma(y+\beta)}{y^{y+\beta-\alpha}}>\frac{e^{x} \Gamma(x+\beta)}{x^{x+\beta-\alpha}}
$$


for $y>x>0$, which can be rearranged as

$$
\begin{gathered}
\frac{\Gamma(y+\beta)}{\Gamma(x+\beta)}>e^{x-y} \frac{y^{y+\beta-\alpha}}{x^{x+\beta-\alpha}} \\
{\left[\left(\frac{y}{x}\right)^{\alpha-\beta} \frac{\Gamma(y+\beta)}{\Gamma(x+\beta)}\right]^{1 /(y-x)}>\frac{1}{e}\left(\frac{y^{y}}{x^{x}}\right)^{1 /(y-x)} .}
\end{gathered}
$$

The proof of Theorem 5 is complete.

\section{REFERENCES}

[1] H. Alzer, On some inequalities for the gamma and psi functions, Math. Comp. 66 (1997), no. $217,373-389$.

[2] G. D. Anderson, R. W. Barnard, K. C. Richards, M. K. Vamanamurthy and M. Vuorinen, Inequalities for zero-balanced hypergeometric functions, Trans. Amer. Math. Soc. 347 (1995), no. $5,1713-1723$.

[3] R. D. Atanassov and U. V. Tsoukrovski, Some properties of a class of logarithmically completely monotonic functions, C. R. Acad. Bulgare Sci. 41 (1988), 21-23.

[4] C. Berg, Integral representation of some functions related to the gamma function, Mediterr. J. Math. 1 (2004), no. 4, 433-439.

[5] Ch.-P. Chen and F. Qi, Logarithmically completely monotonic functions relating to the gamma function, J. Math. Anal. Appl. 321 (2006), no. 1, 405-411.

[6] A. Erdélyi(ed), Higher Transcendental Functions, Vol 1, McGraw-Hill, New York, 1953.

[7] I. S. Gradshteyn and I. M. Ryzhik, Table of Integrals, Series and Products, 6th Ed., Academic Press, New York, 2000.

[8] A. Z. Grinshpan and M. E. H. Ismail, Completely monotonic functions involving the Gamma and q-gamma functions, Proc. Amer. Math. Soc. 134 (2006), 1153-1160.

[9] B.-N. Guo, Y.-J. Zhang and F. Qi, Refinements and sharpenings of some double inequalities for bounding the gamma function, J. Inequal. Pure Appl. Math. 9 (2008), no. 1, Art. 17; Available online at http://jipam.vu.edu .au/article.php?sid=953.

[10] S. Guo, Some classes of completely monotonic functions involving the gamma function, Internat. J. Pure Appl. Math. 30 (2006), no. 4, 561-566.

[11] S. Guo, F. Qi, and H. M. Srivastava, Necessary and sufficient conditions for two classes of functions to be logarithmically completely monotonic, Integral Transforms Spec. Funct. 18 (2007), no. 11, 819-826.

[12] S. Guo, F. Qi, and H. M. Srivastava, Supplements to a class of logarithmically completely monotonic functions associated with the gamma function, Appl. Math. Comput. 197 (2008), no. 2, 768-774.

[13] M. E. H. Ismail, L. Lorch, and M. E. Muldoon, Completely monotonic functions associated with the gamma function and its q-analogues, J. Math. Anal. Appl. 116 (1986), 1-9.

[14] M. E. H. Ismail and M. E. Muldoon, Inequalities and monotonicity properties for gamma and q-gamma functions, in: R.V.M. Zahar (Ed.), Approximation and Computation: A Festschrift in Honour of Walter Gautschi, ISNM, Vol. 119, BirkhRauser, Basel, 1994, 309-323.

[15] J. D. Kečlić and P. M. Vasić, Some inequalities for the gamma function, Publ. Inst. Math. (Beograd) (N. S.) 11 (1971), 107-114.

[16] M. Merkle, Gurland's ratio for the gamma function, Comput. Math. Appl. 49 (2005), 389-406.

[17] M. E. Muldoon, Some monotonicity properties and characterizations of the gamma function, Aequationes Math. 18 (1978), 54-63.

[18] F. Qi, An integral expression and some inequalities of Mathieu type series, Rostock. Math. Kolloq. 58 (2004), 37-46.

[19] F. Qi, Integral expression and inequalities of Mathieu type series, RGMIA Res. Rep. Coll. 6 (2003), no. 2, Art. 10; Available online at http://www.staff.vu.edu.au/rgmia/v6n2.asp.

[20] F. Qi, Three classes of logarithmically completely monotonic functions involving gamma and psi functions, Integral Transforms Spec. Funct. 18 (2007), no. 7, 503-509.

[21] F. Qi and Ch.-P. Chen, A complete monotonicity property of the gamma function, J. Math. Anal. Appl. 296 (2004), no. 2, 603-607.

[22] F. Qi, Ch.-P. Chen, and B.-N. Guo, Notes on double inequalities of Mathieu's series, Internat. J. Math. Math. Sci. 2005 (2005), no. 16, 2547-2554.

[23] F. Qi and B.-N. Guo, Complete monotonicities of functions involving the gamma and digamma functions, RGMIA Res. Rep. Coll. 7 (2004), no. 1, Art. 8, 63-72; Available online at http://www.staff.vu.edu.au/rgmia/v7n1.asp. 
[24] F. Qi, B.-N. Guo and Ch.-P. Chen, Some completely monotonic functions involving the gamma and polygamma functions, J. Aust. Math. Soc. 80 (2006), 81-88.

[25] Y.-J. Wei, S.-L. Zhang, and Ch.-P. Chen, Logarithmically completely monotonic functions and Gurland's ratio for the gamma function, Adv. Stud. Contemp. Math. 15 (2007), no. 2, 253-257.

(S. Guo) Department of Mathematics, Zhongyuan University of Technology, Zhengzhou City, Henan Province, 450007, China

E-mail address: sguo@hotmail.com, senlinguo@gmail.com

(F. Qi) Research Institute of Mathematical Inequality Theory, Henan Polytechnic University, Jiaozuo City, Henan Province, 454010, China

E-mail address: qifeng618@gmail.com, qifeng618@hotmail.com, qifeng618@qq.com

$U R L:$ http://qifeng618.spaces.live.com 